\title{
Quantifying cerebral blood flow: regional regulation with global implications
}

\author{
Scott A. Small
}

Columbia University, College of Physicians and Surgeons, New York, New York, USA.

In 1948, Seymour S. Kety and Carl F. Schmidt published back-to-back papers in the JCI that are widely acknowledged as landmarks. Upon publication, the studies resolved a century-old debate, irrefutably demonstrating that cerebral blood flow is regionally regulated. The reported findings turned out to be so powerful in their implications that they provided the inspirational spark that illuminated a brand-new field: functional brain imaging. Thus these papers are landmarks of the rarest kind, not only ending a controversy, but also giving birth to one of the most exciting fields within modern day neuroscience.

During the 19th century, vascular physiologists began to show that blood flow to an organ is not solely regulated by extrinsic factors like cardiac output. Rather, a growing number of studies suggested that blood flow could be regulated intrinsically within the organ itself $(1,2)$. Chemically induced vascular dilatation was thought to underlie the intrinsic effect, such that regional expansion of vascular volume would then cause a subsequent increase in blood flow. Although this was proposed as a general mechanism, a controversy arose as to whether it applied in the unique case of cerebral blood flow (CBF). The prevailing view was that, because of the rigid skull, even subtle increases in cerebral volume would cause untenable elevations in intracranial pressure. This gave rise to a strong position, one that fired a centurylong debate: that $\mathrm{CBF}$ cannot be regulated intrinsically and that any change in CBF must occur via extrinsic mechanisms such as changes in systemic blood pressure or cardiac output (3).

Those in the minority clung to the view that local regulation of CBF may occur, although admittedly this was based more on theoretical considerations then on empirical evidence. Toward the end of the 19th century, Charles S. Roy and Charles S. Sherrington provided the first evidence supporting this position (4). In their experiments, a novel monitoring

Nonstandard abbreviations used: BOLD, blood oxygen level-dependent; CBF, cerebral blood flow; fMRI, functional magnetic resonance imaging.

Conflict of interest: The author has declared that no conflict of interest exists.

Citation for this article: J. Clin. Invest. 114:1046-1048 (2004). doi:10.1172/JCI200423159. device was placed on the brain surface of anesthetized dogs - essentially measuring fluctuations in volume - and shifts in intracranial volume were observed during chemical challenges. These fluctuations were presumed to reflect a change in vascular volume and therefore to represent a proxy measure of changing $\mathrm{CBF}$. Nevertheless, this paper did not resolve the controversy of whether intrinsic or only extrinsic factors regulated CBF. Advocates of the opposition view had an easy time criticizing the paper, and much of this criticism was legitimate (5). For example, as critics pointed out, the monitoring device was only an indirect indicator of vascular volume and, in any case, the yielded measurements were not quantitative.

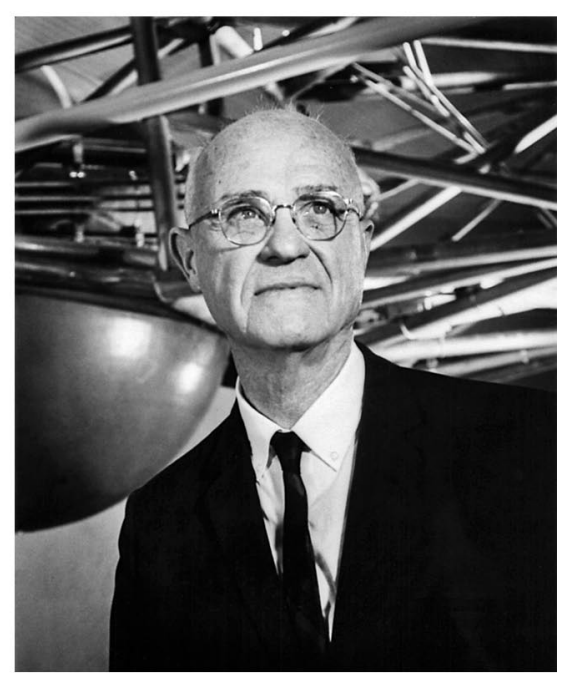

Figure 1

Carl F. Schmidt (left; image courtesy of the National Library of Medicine) and Seymour S. Kety (right).

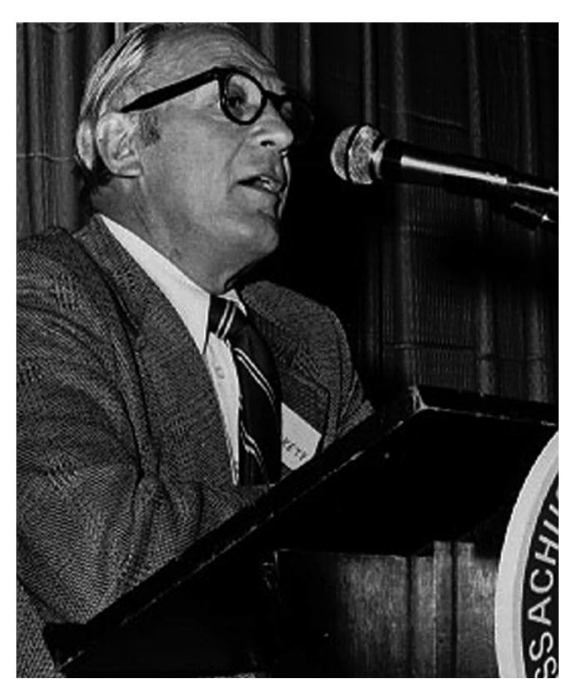

Or, as was also noted, the experiments were performed in anesthetized subjects and required an invasive craniectomy, and so the observed effects did not necessarily reflect the natural environment (5). In fact, the debate over whether intrinsic factors can regulate CBF raged on for over 60 years more, until the 1948 JCI publications by Kety and Schmidt (Figure 1).

During his medical training, Kety became familiar with Fick's principle and its successful application in quantifying cardiac blood flow (6). Fick's principle states that blood flow to an organ must equal the rate in which the organ metabolizes a blood constituent, divided by the constituent's vascular concentration - its arterial-venous difference. Defying the conventional approach, which relied on the endogenous gas oxygen, Kety had subjects inhale the exogenous gas nitrous oxide (Figure 2) (7). Kety realized that, because nitrous oxide is metabolically inert (relatively speaking), he could simplify Fick's principle so that instead of three variables there were two and that, by measuring the arterial-venous concentration of nitrous oxide, he could solve the equation for CBF. In so doing, and by paying fastidi- 

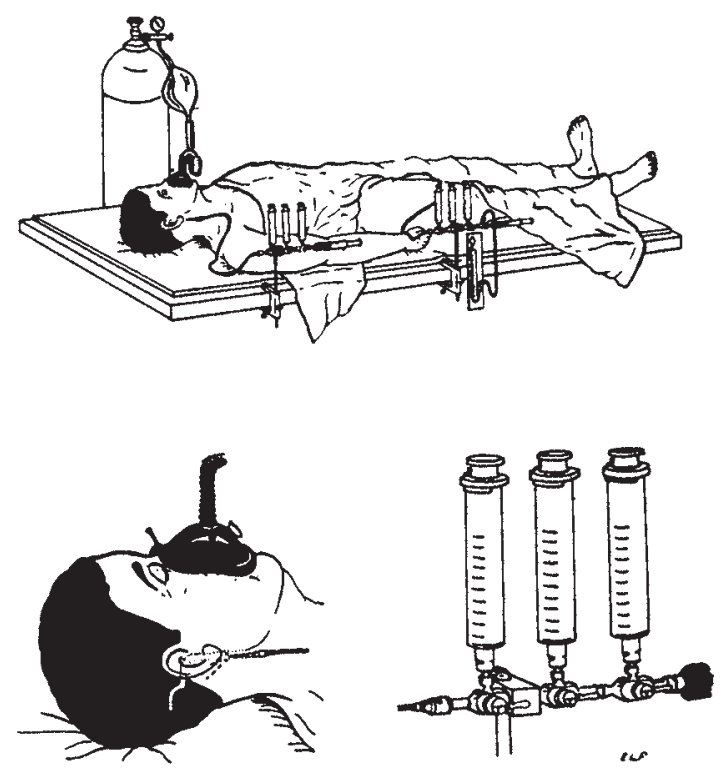

Figure 2

Experimental set-up for cerebral blood flow determination. Position of needles in internal jugular is shown as well as plastic tubing, manifolds, sampling and flushing syringes, and inhalation system. Mean arterial blood pressure is read from a mercury manometer attached to the arterial manifold. Only the expiratory valve on the mask is shown, the inspiratory valve is between the fluted tubing and the mask. Drawings by Dr. E. L. Foltz. Figure and legend reprinted from ref. 7.

ous attention to technical details, Kety and Schmidt introduced, in their first JCI paper, a technique that, for the first time ever, generated a quantitative measure of CBF noninvasively in unanesthetized humans. As if this were not enough, in the same paper they then exploited $\mathrm{CBF}$ and the measurements of arterial-venous blood gases to quantify cerebral metabolic rates of oxygen consumption - again a first-time achievement in the conscious human.

Although a technical tour de force, the first paper simply set the stage for the decisive findings presented in the second paper (8). In this definitive paper, Kety and Schmidt used the innovative nitrous oxide approach to show that inhaled carbon dioxide or oxygen has a predictable affect on CBF (Figure 3). Most importantly, they established that mechanisms intrinsic to the brain must regulate the effect, finally and forevermore laying to rest the controversy surrounding CBF regulation.

At the time of these publications, Schmidt and even Kety were considered vascular physiologists more than brain scientists. Nevertheless, the ability to measure CBF, a proven correlate of brain metabolism, opened up the remarkable possibility of studying brain function in humans. Although correlating behavior defects with structural lesions detected after death had its successes, this approach severely limited the ability to localize brain function and disease states in a systematic fashion. Thus, Kety and Schmidt's approach brought with it the tantalizing realization that mapping brain dysfunction in living subjects was within reach. Of course, a single measure that reflects the $\mathrm{CBF}$ of the whole brain was inadequate for investigating brain function in all its complexity. The next goal became obvious: Build on these primary findings to develop techniques that can record $\mathrm{CBF}$ from many individual brain regions.

During subsequent decades, responding directly to this challenge and exploiting many of the principles introduced in these landmark papers (6), researchers ultimately met this goal. The effort was spearheaded by Kety's own work published during the 1950s - yet more landmarks for this unstoppable investigatorand through a community-wide series of technical achievements. A string of chemical tracers was identified with which to estimate regional $\mathrm{CBF}$ and, through sheer technical wizardry, an assembly line of "brain cameras" was introduced that kept on improving the spatial resolution with which these tracers could be visualized. Thus, conceived out of Kety and Schmidt's landmark papers, the field of functional brain imaging was born.
As a field, functional brain imaging has maintained its innovative spirit, unveiling its latest technique, functional magnetic resonance imaging (fMRI), during the 1990s. Although developed a half century later, even fMRI owes much of its intellectual heritage to Kety and Schmidt's original papers. For example, one version of FMRI relies on the endogenous tracer deoxyhemoglobin - the blood oxygen level-dependent (BOLD) signal- to estimate regional metabolism (9). Here again, a modified version of Fick's principle is invoked to account for why deoxyhemoglobin correlates with CBF and oxygen metabolism. fMRI presents many advantages over earlier techniques, including superior spatial and temporal resolution, greater safety to the subject, and easier use for the experimenter. Because of these attractions, psychologists in particular have flocked to BOLD AMRI in their efforts to map normal brain function.

Nevertheless, in the shadow of the high standards set by Kety and Schmidt, AMRI still needs to be perfected. In contrast to functional measures generated by other imaging techniques - such as positron emission tomography or single photon emission computerized tomography - the BOLD signal is

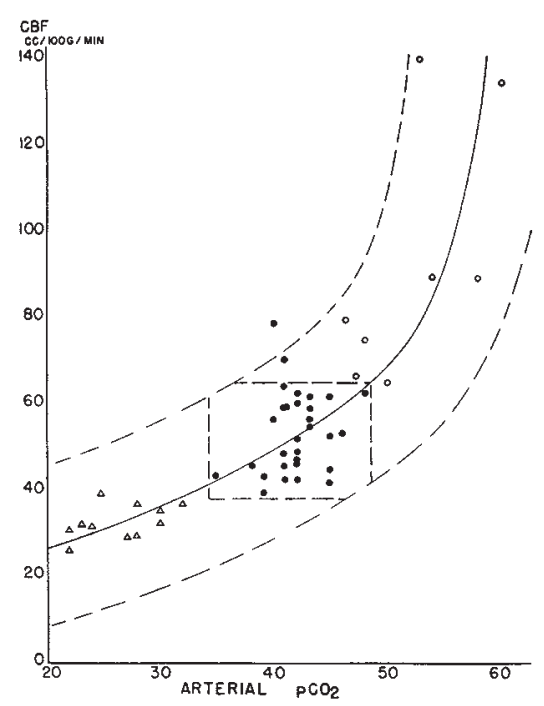

Figure 3

The relationship between cerebral blood flow and arterial $\mathrm{CO}_{2}$ tension. Arterial $\mathrm{CO}_{2}$ tension was varied from the normal (filled circles) by hyperventilation (triangles) or by inhalation of $5-7 \% \mathrm{CO}_{2}$ (open circles). The broken curves bound $98 \%$ of the observations while the central polygon encloses $94 \%$ of the normal values. Figure reprinted and legend adapted from ref. 8. 
unfortunately not quantitative. As stated explicitly in the title of Kety and Schmidt's first JCI paper, generating a quantifiable measure was a key achievement of their original efforts. The absence of quantification is one of the main reasons that BOLD fMRI has yet to make significant contributions to clinical neuroscience, such as reliably mapping neurologic or psychiatric illness. It is this potential clinical utility, one that emerged from Kety's lifelong interests, that motivates the current crop of fMRI investigators. The combination of innovation and rigor that characterized Kety and Schmidt's landmark papers serves as a model to us all.
Address correspondence to: Scott A. Small, Columbia University, College of Physicians and Surgeons, 630 West 168th Street, New York, New York 10032, USA. Phone: (212) 305-9194; Fax: (212)342-2849; E-mail: sas68@columbia.edu.

1. Roy, C.S., and Brown, J.G. 1879. The blood pressure and its variation in the arterioles, capillaries and smaller veins. J. Physiol. 2:323-359.

2. Gaskell, T.W.H. 1880. On the tonicity of the heart and blood vessels. J. Physiol. 3:38-75

3. Hill, L. 1896. The physiology and pathology of the cerebral circulation: an experimental research. J. \& A. Churchill. London, United Kingdom. 208 pp.

4. Roy, C.S., and Sherrington, C.S. 1890 . On the regulation of the blood supply of the brain. J. Physiol. 11:85-108.
5. Friedland, R.P., and Iadecola, C. 1991. Roy and Sherrington (1890): a centennial reexamination of "On the regulation of the blood-supply of the brain”. Neurology. 41:10-14.

6. Sokoloff, L. 2000. In memoriam: Seymour Kety, M.D. 1915-2000. J. Cereb. Blood Flow Metab. 20:1271-1275

7. Kety, S.S., and Schmidt, C.F. 1948. The nitrous oxide method for the quantitative determination of cerebral blood flow in man: theory, procedure and normal values. J. Clin. Invest. 27:476-483.

8. Kety, S.S., and Schmidt, C.F. 1948. The effects of altered arterial tensions of carbon dioxide and oxygen on cerebral blood flow and cerebral oxygen consumption of normal young men. J. Clin. Invest. 27:484-492.

9. Ogawa, S., Lee, T.M., Nayak, A.S., and Glynn, P. 1990. Oxygenation-sensitive contrast I magnetic resonance image of rodent brain at high magnetic fields. Magn. Reson. Med. 14:68-78.

\section{The legacy of Homer W. Smith: mechanistic insights into renal physiology}

\section{Gabriel Navar}

Department of Physiology and Hypertension and Renal Center of Excellence, Tulane University Health Sciences Center, New Orleans, Louisiana, USA.

\begin{abstract}
In 1945, Homer W. Smith published an article in the JCI that clearly demonstrated that para-aminohippuric acid is the most suitable agent for the evaluation of renal plasma flow in both humans and dogs; in addition, the paper provided detailed methodology that is still in use today. This paper is but one of many outstanding works performed by Smith and his colleagues that clearly established the clearance technique as a powerful noninvasive approach to gain mechanistic insights into intrarenal function.
\end{abstract}

In light of today's practices by many prestigious journals seeking the holy grail of impact factor, it is entirely possible that Homer W. Smith (Figure 1), legendary renal physiologist and prolific author of numerous publications in the Journal of Clinical Investigation during the 1930s and '40s, would have been given a polite but firm rejection of one of the most cited articles that has been published in the JCI (1). "Dear Dr. Smith," the letter would say, "the Editorial Board regrets to inform you that it feels that your paper would be more appropriate for a specialized journal of kidney research; in addition, it was felt that your paper is primarily descriptive in nature." In reality, this highly-cited paper $(2,407$ times as of this writing) by Smith and his associates is a meticulously docu-

Nonstandard abbreviations used: $\mathrm{PAH}$, para-aminohippuric acid.

Conflict of interest: The author has declared that no conflict of interest exists.

Citation for this article: J. Clin. Invest. 114:1048-1050 (2004). doi:10.1172/JCI200423150. mented - albeit descriptive - work evaluating the differences among a number of organic acids that are excreted by the kidney with very high efficiency because they undergo tubular secretion (1).

This paper is but one of a long string of studies by Smith's laboratory group during the 1930s and '40s. Smith and his associates were incredibly prolific and contributed greatly to the development of many important concepts in renal physiology, in particular those related to the use of the clearance technique to determine how specific substances are handled by the kidneys (2). His efforts to integrate knowledge in renal physiology are readily reflected in his monumental work, entitled The kidney: structure and function in bealth and disease, published in 1951 (2). His outstanding contributions to the world of renal physiology have been noted by many of his trainees and documented in the book published by Chasis and Goldring in 1965 (3). The book includes, in addition to several of his speeches and essays, talks given in honor of Smith. In the words of
S.E. Bradley at a dinner in 1962, "Homer Smith and the school Homer Smith has nurtured have been responsible for the major discoveries that link New York University and the kidney in men's minds the world over." Bradley also reflected on the insatiable curiosity of Smith in recounting when, as a young renal physiologist in Charlottesville, Virginia, Smith generated attention by following circus camels, pot in hand, so that he could collect their urine and try to understand how these animals could excrete such concentrated urine.

In his address before the National Academy of Sciences shortly after Smith's death in 1962, R.F. Pitts eloquently placed in perspective his extraordinary contributions. "Smith was the first to understand what renal clearance really meant and to appreciate what a powerful tool the clearance concept could be if properly justified and applied ... For over thirty years, he dominated his chosen field in a way that few, if any, have dominated other fields. His personal investigations, his broad and inclusive concepts, the methods of study of function which he developed and popularized, the texts that he wrote, and the many investigators and students of medicine whom he trained and influenced established him as the acknowledged master of all things renal."

A strong advocate of the importance of collaborations between preclinical and clinical departments, Smith was a driving 